\title{
Equalizers and coactions of groups
}

\author{
by \\ Martin Arkowitz (Hanover, NH) \\ and Mauricio Gutierrez (Medford, MA)
}

\begin{abstract}
If $f: G \rightarrow H$ is a group homomorphism and $p, q$ are the projections from the free product $G * H$ onto its factors $G$ and $H$ respectively, let the group $\mathcal{E}_{f} \subseteq G * H$ be the equalizer of $f p$ and $q: G * H \rightarrow H$. Then $p$ restricts to an epimorphism $p_{f}=$ $p \mid \mathcal{E}_{f}: \mathcal{E}_{f} \rightarrow G$. A right inverse (section) $G \rightarrow \mathcal{E}_{f}$ of $p_{f}$ is called a coaction on $G$. In this paper we study $\mathcal{E}_{f}$ and the sections of $p_{f}$. We consider the following topics: the structure of $\mathcal{E}_{f}$ as a free product, the restrictions on $G$ resulting from the existence of a coaction, maps of coactions and the resulting category of groups with a coaction and associativity of coactions.
\end{abstract}

1. Introduction. The notion of an action of one group on another has been studied extensively. In general terms this consists of a homomorphism $G \times H \rightarrow G$ whose restriction to $G$ is the identity homomorphism and whose restriction to $H$ is a fixed homomorphism $H \rightarrow G$. The dual concept of a coaction is given by a homomorphism $G \rightarrow G * H$ (the free product of $G$ and $H$ ) whose compositions with the projections of $G * H$ onto $G$ and $H$ are the identity homomorphism id and a fixed homomorphism $f: G \rightarrow H$, respectively. The motivation for studying coactions is two-fold. First of all, in the special case $G=H$ and $f=\mathrm{id}$, a coaction is just a comultiplication of the group $G$. This is a basic notion which has been considered by several authors ([7], [5], [1]). Coactions are natural generalizations of group comultiplications. Secondly, coactions have been widely studied in the context of algebraic topology. If $g: X \rightarrow Y$ is a map of spaces and $C_{g}$ is the mapping cone of $g$, then there is a coaction of the suspension $\Sigma X$ on $C_{g}$ given by a map $C_{g} \rightarrow C_{g} \vee \Sigma X$. This has proved to be an extremely useful tool in homotopy theory (see $[6$, Chs. 11,14$]$ and $[9$, Ch. 2]). Coactions of groups are the natural analogues of these topological coactions. Moreover, in a future paper we hope to study functors from the homotopy category to

2000 Mathematics Subject Classification: Primary 20E06; Secondary 20 F99.

Key words and phrases: free products, equalizers, coactions. 
the category of groups (such as the fundamental group functor) which carry topological coactions to group coactions.

In this paper we continue our investigation of group coactions which was begun in [2]. However, our approach here is different from that of [2]. We consider a fixed homomorphism $f: G \rightarrow H$ and the projections $p: G * H \rightarrow G$ and $q: G * H \rightarrow H$ and define $\mathcal{E}_{f}$ to be the equalizer of $f p$ and $q$. Then $p \mid \mathcal{E}_{f}: \mathcal{E}_{f} \rightarrow G$ is a homomorphism, and we easily see that a coaction of $H$ on $G$ (relative to $f$ ) is just a section $s: G \rightarrow \mathcal{E}_{f}$ of $p \mid \mathcal{E}_{f}$. All of the questions about coactions which we investigate here are very conveniently expressed and discussed in terms of sections into the equalizer. In a different form or in special cases such sections have been considered in [1]-[5] and [7].

In this paper we investigate three natural topics related to $\mathcal{E}_{f}$. The first (in $\S \S 3$ and 5) is the structure of $\mathcal{E}_{f}$ with special emphasis on its free factor decompositions. We show that $\mathcal{E}_{f}$ is the free product of $\operatorname{ker} f$ and a free group. We also investigate subgroups $A \subseteq G$ such that $\mathcal{E}_{f \mid A}$ is a free factor of $\mathcal{E}_{f}$. If $A \cdot \operatorname{ker} f=G$ or if $A \cap \operatorname{ker} f=1$ then we prove that this always holds.

Our second topic ( $\S \S 4$ and 5) concerns the restrictions on $G$ which result from the existence of a coaction rel $f$. A classical result in [5] and [7] states that in the case $G=H$ and $f=\mathrm{id}$, the identity map, $G$ admits a section $G \rightarrow \mathcal{E}_{\text {id }}$ (called a comultiplication) if and only if $G$ is free. We extend this result by showing that $G$ admits a coaction rel $f$ if and only if $G$ is the free product of a subgroup of ker $f$ and a free group. Also, if $A \subseteq G$ and $G$ admits a coaction $s$ such that $s(A) \subseteq \mathcal{E}_{f \mid A}$, then $A$ is a free factor of $G$ if and only if $\mathcal{E}_{f \mid A}$ is a free factor of $\mathcal{E}_{f}$.

Our final topic deals with homomorphisms of coactions. Let $s_{i}$ be a coaction of $H_{i}$ on $G_{i}$ rel $f_{i}(i=1,2)$ and let $\phi_{1}: G_{1} \rightarrow G_{2}$ and $\phi_{2}: H_{1} \rightarrow H_{2}$ be a pair of homomorphisms such that $f_{2} \phi_{1}=\phi_{2} f_{1}$. Then $\phi_{1} * \phi_{2}: G_{1} * H_{1} \rightarrow$ $G_{2} * H_{2}$ induces a homomorphism $\Phi: \mathcal{E}_{f_{1}} \rightarrow \mathcal{E}_{f_{2}}$. If $\Phi s_{1}=s_{2} \phi_{1}$, we call $\left(\phi_{1}, \phi_{2}\right)$ a coaction map $\left(G_{1}, s_{1}\right) \rightarrow\left(G_{2}, s_{2}\right)$, and say that $s_{1}$ induces $s_{2}$. Then we consider the following questions in $\S 6$ : Given $\Phi$ as before, and a coaction $s_{1}$ on $G_{1}$, when does it induce a coaction $s_{2}$ on $G_{2}$ ? Conversely, when is a given coaction $s_{2}$ induced by some $s_{1}$ ?

2. Preliminaries. In this section we present notation and basic facts in group theory. All groups are written multiplicatively. If $G$ is a group, the identity is denoted by $1 \in G$ and the inverse of an element $g \in G$ is written $\bar{g}$ or $g^{-1}$. For $g, g^{\prime} \in G$, the commutator $\left[g, g^{\prime}\right]$ is $g g^{\prime} \bar{g} \bar{g}^{\prime}$. If $S \subseteq G$ is a subset of $G$, then $\langle S\rangle$ is the subgroup generated by $S$. For subsets $S, T$ of $G,[S, T]$ denotes the subgroup generated by all commutators $[s, t]$ with $s \in S, t \in T$. Furthermore, $S-T$ is the set-theoretic difference, i.e., all elements of $S$ which are not in $T$. If $T$ consists of a single element $t$, we write this as $S-t$. 
A subset $X$ of $G$ is independent if $\langle X\rangle$ is a free group with basis $X$. If $G$ and $H$ are groups, the free product $G * H$ is defined in the usual way and the projections are $p: G * H \rightarrow G$ and $q: G * H \rightarrow H$. Moreover, homomorphisms $f: G \rightarrow G^{\prime}$ and $g: H \rightarrow H^{\prime}$ define a homomorphism $f * g$ : $G * H \rightarrow G^{\prime} * H^{\prime}$. The homomorphisms $p$ and $q$ determine a homomorphism $p \times q: G * H \rightarrow G \times H$ into the cartesian product.

LEMma 2.1. The following sequence is exact:

$$
1 \rightarrow[G, H] \rightarrow G * H \stackrel{p \times q}{\longrightarrow} G \times H \rightarrow 1,
$$

and the subgroup $[G, H]$ of $G * H$ is a free group with basis $\{[g, h] \mid g \in$ $G-1, h \in H-1\}$.

The exactness of the sequence is easily proved and the assertion about $[G, H]$ is shown in [8, p. 196, Exercises 23, 24].

When $G=H$, we use $G^{\prime}$ and $G^{\prime \prime}$ to denote the first and second factors of $G * G$ and the projections are written $p^{\prime}$ and $p^{\prime \prime}$ instead of $p$ and $q$. On occasion, we write $g^{\prime} h^{\prime \prime} \in G * H$ or even $g^{\prime} h^{\prime \prime} k^{\prime \prime \prime} \in G * H * H$, for $g \in G$ and $h, k \in H$. A subgroup $A \subseteq G$ is a free factor if there is a subgroup $B$ such that $G=A * B$. It is easy to see that if the group $G$ is free, then this definition and the definition of a free factor in $[7$, p. 113, Ex. 8] coincide.

The identity homomorphism of the group $G$ is denoted by $\operatorname{id}_{G}: G \rightarrow G$ or just by id. The trivial homomorphism $G \rightarrow H$ that carries all of $G$ to $1 \in H$ is denoted by $1: G \rightarrow H$. A set-theoretic section of a homomorphism $f: G \rightarrow H$ is a function $s: H \rightarrow G$ such that $f s=\mathrm{id}_{H}$. If $s$ is a homomorphism, we call it a section. Now suppose that $f_{i}: G_{i} \rightarrow H_{i}, i=1,2$, are homomorphisms. Then a map of homomorphisms $\Phi=\left(\phi_{1}, \phi_{2}\right)$ consists of homomorphisms $\phi_{1}: G_{1} \rightarrow G_{2}$ and $\phi_{2}: H_{1} \rightarrow H_{2}$ such that the following diagram commutes:

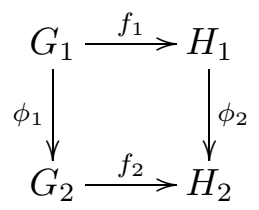

We say that $\Phi=\left(\phi_{1}, \phi_{2}\right)$ is a map of $f_{1}$ to $f_{2}$ and write $\Phi: f_{1} \rightarrow f_{2}$.

The following result, whose proof is straightforward, will be used repeatedly.

Lemma 2.3. Suppose $X$ and $Y$ are disjoint sets whose union is a basis of a free group $G$. If for every $x \in X$, we choose $y_{x} \in Y$, then the set $\left\{x \bar{y}_{x} \mid x \in X\right\} \cup Y$ is a basis of $G$.

3. Equalizers. Let $f: G \rightarrow H$ be a fixed homomorphism and $p$ : $G * H \rightarrow G, q: G * H \rightarrow H$ the two projections. 
Definition 3.1. The equalizer $\mathcal{E}_{f}$ of $f$ is the subgroup $\{w \mid w \in G * H$, $f p(w)=q(w)\}$ of $G * H$ (see [2]). The homomorphisms $p$ and $q$ induce homomorphisms, also denoted by $p$ and $q$, from $\mathcal{E}_{f}$ to $G$ and $H$. For every $g \in G$, denote by $\eta_{g}$ the element $g f(g) \in \mathcal{E}_{f}$.

It is obvious that the group $[G, H]$ is contained in $\mathcal{E}_{f}$. If $G=H$ and $f=\mathrm{id}$, we denote by $\mathcal{E}_{f}$ by $E_{G}$ and $\eta_{g}$ is denoted by $\xi_{g}=g^{\prime} g^{\prime \prime} \in E_{G}$. It is well known ([5], [1]) that $E_{G}$ is a free group with basis $X=\left\{\xi_{g} \mid g \in G-1\right\}$.

Now let $f_{i}: G_{i} \rightarrow H_{i}, i=1,2$, be homomorphisms and $\Phi=\left(\phi_{1}, \phi_{2}\right)$ : $f_{1} \rightarrow f_{2}$ be a map of homomorphisms (2.2). Then $\left(\phi_{1} * \phi_{2}\right) \mid \mathcal{E}_{f_{1}}$ is a homomorphism $\mathcal{E}_{f_{1}} \rightarrow \mathcal{E}_{f_{2}}$, which we also denote by $\Phi$, and the following diagram commutes:

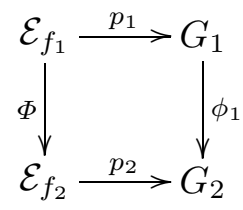

If $f: G \rightarrow H$ is a homomorphism, then we have maps of homomorphisms $F_{2}=\left(\operatorname{id}_{G}, f\right): \operatorname{id}_{G} \rightarrow f$ and $F_{1}=\left(f, \operatorname{id}_{H}\right): f \rightarrow \operatorname{id}_{H}$ and hence homomorphisms

$$
E_{G} \stackrel{F_{2}}{\rightarrow} \mathcal{E}_{f} \stackrel{F_{1}}{\rightarrow} E_{H}
$$

Definition 3.2. The semi-equalizer $E_{f}$ of $f$ is the subgroup $F_{2}\left(E_{G}\right)$ of $\mathcal{E}_{f}$.

It follows immediately that $E_{f}=\left\langle\left\{\eta_{g} \mid g \in G\right\}\right\rangle$ since $F_{2}\left(\xi_{g}\right)=\eta_{g}$.

We adopt the following notation for the remainder of this paper: $K \subseteq G$ is the kernel of $f: G \rightarrow H$ and $I \subseteq H$ is the image of $f$. Consider the group of left cosets $G / K$ and choose a set-theoretic section $\sigma: G / K \rightarrow G$ of the natural epimorphism $\pi: G \rightarrow G / K$ such that $\sigma(1)=1$. We let $C=\left\{\widehat{g}_{j} \mid j \in J\right\}$ be the image of $\sigma$ and hence $C$ is a complete set of coset representatives of $K$ in $G$. For any element $g \in G$ we also denote by $\widehat{g}$ the element $\sigma \pi(g) \in G$, so that $\widehat{g}=\widehat{g}_{j}$ for some $j \in J$. If $D \subseteq C$, we let $E_{D}$ be the subgroup of $E_{f}$ generated by all the $\eta_{\widehat{g}_{j}}$ for $\widehat{g}_{j} \in D$. Note the difference in meaning between $E_{D}$ and $E_{G}$. However, we have:

LEMma 3.3. The homomorphism $F_{1}: \mathcal{E}_{f} \rightarrow E_{H}$ induces an isomorphism $F_{1}^{\prime}: E_{C} \rightarrow E_{I}$.

The proof is straightforward and hence omitted.

Lemma 3.4. The group $E_{G}$ has a basis $B_{1} \cup B_{2} \cup B_{3}$, where $F_{2}\left(B_{1}\right)=1$, $B_{2}=\left\{\xi_{k} \mid k \in K-1\right\}$ and $B_{3}=\left\{\xi_{\widehat{g}_{j}} \mid \widehat{g}_{j} \in C-1\right\}$. Consequently, $E_{f}=K * E_{C}$. 
Proof. Let $B_{1}^{\prime}=\left\{\xi_{g} \mid g \in G-K, g \neq \widehat{g}_{j}, j \in J\right\}$. Then we can write the basis $X=\left\{\xi_{g} \mid g \in G-1\right\}$ of $E_{G}$ as

$$
X=B_{1}^{\prime} \cup B_{2} \cup B_{3} .
$$

By applying Lemma 2.3 twice, we transform $B_{1}^{\prime}$ to

$$
B_{1}=\left\{\xi_{g} \bar{\xi}_{\widehat{g}_{j}} \bar{\xi}_{k} \mid g \in G-K, g \neq \widehat{g}_{j}, g=k \widehat{g}_{j} \text { for some } k \in K-1, j \in J\right\} \text {. }
$$

Then the union of the $B_{i}$ is a basis of $E_{G}$ such that $F_{2}\left(B_{1}\right)=1$. The second statement follows by applying $F_{2}$ to $B_{1} \cup B_{2} \cup B_{3}$.

Next consider the subgroups $[G, H]$ and $[G, I]$ of $G * H$. It is clear that $[G, I] \subseteq \mathcal{E}_{f}$.

Lemma 3.5. If $g, x \in G$ then

$$
[g, f(\bar{x})]=\eta_{g} \bar{\eta}_{x g} \eta_{x}
$$

Consequently, $[G, I] \subseteq E_{f}$.

Proposition 3.6. The following sequence is exact:

$$
1 \rightarrow[G, H] \rightarrow \mathcal{E}_{f} \stackrel{p}{\rightarrow} G \rightarrow 1 .
$$

The proof follows from Lemma 2.1.

We next express the equalizer as a free product.

Proposition 3.7. $\mathcal{E}_{f}=E_{f} *[G, H-I]$.

Proof. The inclusions of $E_{f}$ and $[G, H-I]$ into $\mathcal{E}_{f}$ define a homomorphism $\alpha: E_{f} *[G, H-I] \rightarrow \mathcal{E}_{f}$. We first show that $\alpha$ is one-to-one. It suffices to show that no cancellation can occur in a subword of the form $\left(\eta_{g}^{ \pm 1}\left[g^{\prime}, h\right]^{ \pm 1}\right)^{ \pm 1}$, where $g, g^{\prime} \in G-1$ and $h \in H-I$. But this follows because $h \neq f(\gamma)$ for any $\gamma \in G$ since $h \notin I$. Now we show that $\alpha$ is onto. Let $w \in \mathcal{E}_{f}$ and set $g=p(w)$. Then by Proposition 3.6, $w=\eta_{g} v$ for some $v \in[G, H]$. But $[G, H]=[G, H-I] *[G, I]$ by Lemma 2.1 and $[G, I] \subseteq E_{f}$ by Lemma 3.5. Thus $\alpha$ is onto. This completes the proof.

Corollary 3.8. $\mathcal{E}_{f}=K * E_{C} *[G, H-I]$.

4. Coactions. We begin by recalling and extending some definitions from [2]. If $f: G \rightarrow H$ is a fixed homomorphism, then a (right) coaction rel $f$ of $H$ on $G$ is a homomorphism $s: G \rightarrow G * H$ such that $p s=\operatorname{id}_{G}: G \rightarrow G$ and $q s=f: G \rightarrow H$. A left coaction rel $f$ is similarly defined. We shall only consider right coactions and call them coactions. In the case $G=H$ and $f=\mathrm{id}$, a coaction rel $f$ is called a comultiplication of $G$.

Now let $f_{i}: G_{i} \rightarrow H_{i}$ be homomorphisms, $i=1,2$, and suppose $\Phi=$ $\left(\phi_{1}, \phi_{2}\right): f_{1} \rightarrow f_{2}$ is a map of homomorphisms. 
Definition 4.1. If $s_{i}: G_{i} \rightarrow G_{i} * H_{i}$ are coactions rel $f_{i}$, for $i=1,2$, then $\Phi$ is a coaction homomorphism of $s_{1}$ to $s_{2}$ (written $s_{1} \rightarrow s_{2}$ ) if the following diagram commutes:

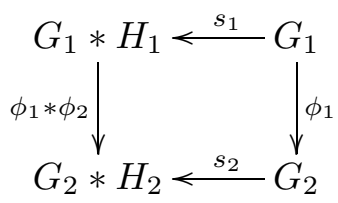

i.e., $\left(\phi_{1}, \phi_{1} * \phi_{2}\right)$ is a map of homomorphisms. In the case $G_{i}=H_{i}, f_{i}=\mathrm{id}$ and $\phi_{1}=\phi_{2}$, a coaction homomorphism is a cohomomorphism of comultiplications as defined in $[1, \S 2]$. Let $s: G \rightarrow G * H$ be a coaction rel $f$ and $A \subseteq G$ a subgroup. If $s(A) \subseteq A * H$, then we say that $A$ is s-stable.

Next we rephrase the definition of coaction, coaction homomorphism and stability in terms of equalizers. This will be the approach we take in subsequent sections.

Let $f: G \rightarrow H$ be a homomorphism, $\mathcal{E}_{f}$ the equalizer of $f$ and $p: \mathcal{E}_{f} \rightarrow G$ the projection. The following result is then obvious.

Lemma 4.2. If $s: G \rightarrow G * H$ is a coaction rel $f$, then $s(G) \subseteq \mathcal{E}_{f}$ and $s: G \rightarrow \mathcal{E}_{f}$ is a section of $p$. Conversely, any section $\sigma: G \rightarrow \mathcal{E}_{f}$ determines a coaction by composing $\sigma$ with the inclusion $\mathcal{E}_{f} \subseteq G * H$. If $s_{i}: G_{i} \rightarrow G_{i} * H_{i}$ are coactions rel $f_{i}, i=1,2$, and $\Phi=\left(\phi_{1}, \phi_{2}\right): f_{1} \rightarrow f_{2}$ is a map of homomorphisms, then $\Phi$ is a coaction homomorphism of $s_{1}$ to $s_{2}$ if and only if the following diagram commutes:

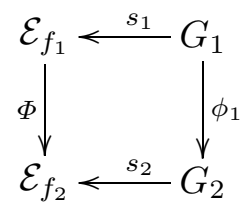

Furthermore, if $s$ is a coaction rel $f$, then $A \subseteq G$ is s-stable if and only if $s(A) \subseteq \mathcal{E}_{f \mid A}$.

We use the same symbol for the coaction $G \rightarrow G * H$ and for the corresponding section $G \rightarrow \mathcal{E}_{f}$.

We conclude this section by characterizing groups that admit a coaction. This was begun in [2, Proposition 4.8] and is also implicit in [3, Lemma 15].

Proposition 4.3. Let $K$ be the kernel of $f: G \rightarrow H$. Then $G$ admits a coaction rel $f$ if and only if $G=N * L$, where $N \subseteq K$ and $L$ is a free group.

Proof. We have proved in [2] that if $G$ admits a coaction then $G=N * L$ as desired. Suppose that $G=N * L$ and that $B$ is a basis for $L$. Define a section $s: G \rightarrow \mathcal{E}_{f}$ of $p$ by $s(x)=x$ if $x \in N$ and $s(b)=\eta_{b}$ for $b \in B$. 
This result should be compared to the analogous one for comultiplications [7] which asserts that a group admits a comultiplication if and only if it is a free group.

5. Stability. In this section we investigate when an $s$-stable subgroup of $G$ is a free factor of $G$. We begin by considering a more general question about equalizers.

Let $f: G \rightarrow H$ be a homomorphism with kernel $K$ and image $I$ and let $A \subseteq G$ be a subgroup. We choose $C_{A}=\{\widehat{x}\}$ to be a complete set of coset representatives of the group $A K / K$, where $\widehat{1}=1$. Because of the isomorphism $A K / K \rightarrow A / A \cap K$ we may assume that $\widehat{x} \in A$. We can extend $C_{A}$ to a complete set $C$ of coset representatives of $G / K$. Then by Lemma 2.1 and Corollary 3.8 we have

$$
\begin{aligned}
\mathcal{E}_{f \mid A} & =(A \cap K) * E_{C_{A}} *[A, H-I] *[A, I-f(A)] \quad \text { and } \\
\mathcal{E}_{f} & =K * E_{C_{A}} * E_{C-C_{A}} *[G-A, H-I] *[A, H-I] .
\end{aligned}
$$

Theorem 5.2. (1) If $A K=G$, then $\mathcal{E}_{f \mid A}$ is a free factor of $\mathcal{E}_{f}$ if and only if $A \cap K$ is a free factor of $K$.

(2) If $A \cap K=1$, then $\mathcal{E}_{f \mid A}$ is a free factor of $\mathcal{E}_{f}$.

Proof. (1) If $A K=G$ then $I-f(A)=\emptyset$. Therefore by (5.1), if $A \cap K$ is a free factor of $K$, then $\mathcal{E}_{f \mid A}$ is a free factor of $\mathcal{E}_{f}$. Conversely, if $\mathcal{E}_{f \mid A}$ is a free factor of $\mathcal{E}_{f}$, then $A \cap K$ is a free factor of $K$ by [3, p. 1543, (20)] since $A \cap K=\mathcal{E}_{f \mid A} \cap K$.

(2) Since $A \cap K=1$, we can choose $C_{A}$ to be $A$ itself and so $\widehat{x}=x$ for all $x \in A$. By (5.1) it suffices to show that $E_{C_{A}} *[A, I-f(A)]$ is a free factor of $\mathcal{E}_{f}$. Now we choose the coset representative set $C$ in a special way: let $\left\{g_{j} \mid j \in J\right\}$ be a complete set of coset representatives of $G / A K$, with 1 representing $A K$. Then the set of all $g_{j} x$ with $j \in J$ and $x \in C_{A}=A$ is a complete set of coset representatives $C$ of $G / K$. We have $C-C_{A}=\left\{g_{j} x \mid\right.$ $\left.g_{j} \neq 1, x \in C_{A}\right\}$. Let $B_{1}=\left\{\eta_{g_{j}} \mid j \in J, g_{j} \neq 1\right\}$ and $B_{2}^{\prime}=\left\{\eta_{g_{j} x} \mid j \in J\right.$, $\left.g_{j} \neq 1, x \neq 1\right\}$ be subsets of $E_{f}$ which generate subgroups $D_{1}=\left\langle B_{1}\right\rangle$ and $D_{2}^{\prime}=\left\langle B_{2}^{\prime}\right\rangle$. Then $E_{C-C_{A}}=D_{1} * D_{2}^{\prime}$. A change of basis in $B_{1} \cup B_{2}^{\prime}$ yields a basis $B_{1} \cup B_{2}$, where

$$
B_{2}=\left\{\bar{\eta}_{g_{j}} \eta_{g_{j} x} \mid j \in J, x \in A, g_{j} \neq 1, x \neq 1\right\}
$$

by Lemma 2.3. Thus, if $D_{2}=\left\langle B_{2}\right\rangle$, we have $E_{C-C_{A}}=D_{1} * D_{2}$.

Now we show $E_{C_{A}} *[A, I-f(A)]=E_{C_{A}} * D_{2}$, which is a free factor of $\mathcal{E}_{f}$. Let $[y, f(g)] \in[A, I-f(A)]$. Then $\bar{g} \equiv g_{j} x$ modulo $K$, for some $x \in A$ and $j \in J$. Note that $g_{j} \neq 1$ since $f(\bar{g}) \notin f(A)$. Therefore $f(g)=f\left(g_{j} x\right)^{-1}$ and by Lemma 3.5 , 


$$
[y, f(g)]=\left[y, f\left(g_{j} x\right)^{-1}\right]=\eta_{y} \bar{\eta}_{g_{j} x y} \eta_{g_{j} x}=\eta_{y}\left(\bar{\eta}_{g_{j}} \eta_{g_{j} x y}\right)^{-1}\left(\bar{\eta}_{g_{j}} \eta_{g_{j} x}\right),
$$

which lies in $E_{C_{A}} * D_{2}$. Thus $E_{C_{A}} *[A, I-f(A)] \subseteq E_{C_{A}} * D_{2}$.

Conversely, if $\bar{\eta}_{g_{j}} \eta_{g_{j} x} \in B_{2}$, then by Lemma 3.5 ,

$$
\bar{\eta}_{g_{j}} \eta_{g_{j} x}=\left[x, f\left(\bar{g}_{j}\right)\right]^{-1} \eta_{x}
$$

which is in $E_{C_{A}} *[A, I-f(A)]$, and this proves the other inclusion.

The following corollary is now clear.

Corollary 5.3. Let $s: G \rightarrow G * H$ be a coaction rel $f$. Assume that $A \subseteq G$ is an s-stable subgroup.

(1) If $A K=G$, then $A$ is a free factor of $G$ if and only if $A \cap K$ is a free factor of $K$.

(2) If $A \cap K=1$, then $A$ is a free factor of $G$.

REMARK 5.4. Part (2) of the preceding corollary was proved in [2, Theorem 3.7] in the special case where $K=\operatorname{ker} f=1$ and $I=\operatorname{im} f$ is a free factor of $H$. In particular, if $m: G \rightarrow G * G$ is a comultiplication and $A \subseteq G$ is $m$-stable, then $A$ is a free factor of $G$.

Even if $A \cap K$ is a free factor of $G$ in Theorem 5.2 above, $\mathcal{E}_{f \mid A}$ may not be a free factor of $\mathcal{E}_{f}$, as the following example shows.

Example 5.5. Suppose $K_{0}=\langle x\rangle$ and $L=\langle y\rangle$ are infinite cyclic groups. Let $G=K_{0} * L, H=L$ and $f: G \rightarrow H$ be defined by $f(x)=1, f(y)=y$. Finally, let $A=\left\langle x, y^{2}\right\rangle \subseteq G$. Thus $K=\operatorname{ker} f$ is the free product of all the $y^{i} K_{0} y^{-i}, i \in \mathbb{Z}$, and $K \cap A$ is the free product of the $y^{i} K_{0} y^{-i}$ for $i$ even, and so $K \cap A$ is a free factor of $K$. However, $\mathcal{E}_{f \mid A}$ is not a free factor of $\mathcal{E}_{f}$. To see this let $Z=\langle z\rangle$ be an infinite cyclic group and define the map $r: A * H \rightarrow Z$ by $r\left(x^{\prime}\right)=1, r\left(\left(y^{\prime}\right)^{2}\right)=z$ and $r\left(y^{\prime \prime}\right)=1$. This induces a map $r: \mathcal{E}_{f \mid A} \rightarrow Z$ which maps $\eta_{y^{2}}$ to $z$. Then any extension $\widetilde{r}$ of $r$ to $\mathcal{E}_{f}$ must send $\eta_{y}^{2}$ to $z$ because

$$
\widetilde{r}\left(\eta_{y}^{2}\right)=\widetilde{r}\left(y^{\prime} y^{\prime \prime} y^{\prime} y^{\prime \prime}\right)=\widetilde{r}\left(y^{\prime}\right) \widetilde{r}\left(y^{\prime}\right)=r\left(\left(y^{\prime}\right)^{2}\right)=z,
$$

and this is a contradiction (cf. [2, Example 7.3]).

6. Compatibility. Assume we are given coactions $s_{i}: G_{i} \rightarrow \mathcal{E}_{f_{i}}$ and a map of homomorphisms $\Phi=\left(\phi_{1}, \phi_{2}\right): f_{1} \rightarrow f_{2}$. In this section we consider the question of when $\Phi$ is a coaction homomorphism $s_{1} \rightarrow s_{2}$. More precisely, we let $\Phi$ and one of the two coactions be fixed and ask whether the other exists so that $\Phi$ is a coaction homomorphism. The notation $\Phi=\left(\phi_{1}, \phi_{2}\right)$ holds throughout this section.

Definition 6.1. Suppose $f_{i}: G_{i} \rightarrow H_{i}, i=1,2$, are homomorphisms and $\Phi: f_{1} \rightarrow f_{2}$ is a map of homomorphisms. Let $s_{1}: G_{1} \rightarrow \mathcal{E}_{f_{1}}$ be a 
coaction; if there exists a coaction $s_{2}: G_{2} \rightarrow \mathcal{E}_{f_{2}}$ such that $\Phi$ is a coaction homomorphism $s_{1} \rightarrow s_{2}$, then we say that $s_{1}$ and $s_{2}$ are compatible. Given a coaction $s_{2}$, if there exists a coaction $s_{1}$ so that $\Phi$ is a coaction homomorphism $s_{1} \rightarrow s_{2}$, we also say that $s_{1}$ and $s_{2}$ are compatible. In the former case we say $s_{1}$ induces $s_{2}$, and in the latter, $s_{2}$ restricts to $s_{1}$.

By Proposition 3.6 we have the commutative diagram with exact rows

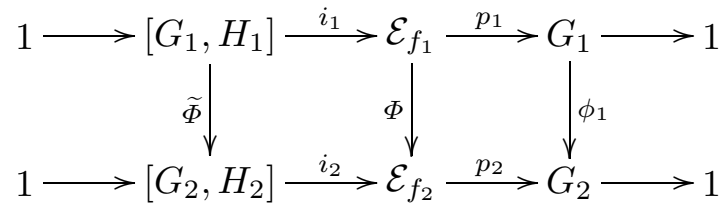

where $i_{1}$ and $i_{2}$ are inclusions and $\widetilde{\Phi}$ is the restriction of $\Phi$. Assume that $G_{1}$ is free with basis $B$ and let $s_{i}$ be coactions as above. We first define a homomorphism $D_{B}^{\prime}\left(s_{1}, s_{2}\right): G_{1} \rightarrow \mathcal{E}_{f_{2}}$ by

$$
D_{B}^{\prime}\left(s_{1}, s_{2}\right)(b)=\Phi\left(s_{1}(b)\right)\left(s_{2} \phi_{1}(\bar{b})\right)
$$

for every $b \in B$. Then $p_{2} D_{B}^{\prime}\left(s_{1}, s_{2}\right)=1$ and so $D_{B}^{\prime}\left(s_{1}, s_{2}\right)$ factors through $\left[G_{2}, H_{2}\right]$.

Definition 6.2. If $G_{1}$ is free with basis $B$, then the homomorphism $D_{B}\left(s_{1}, s_{2}\right): G_{1} \rightarrow\left[G_{2}, H_{2}\right]$ defined by $i_{2} D_{B}\left(s_{1}, s_{2}\right)=D_{B}^{\prime}\left(s_{1}, s_{2}\right)$ is called the difference homomorphism of $s_{1}$ and $s_{2}$. If $f: G \rightarrow H$ and $G$ is free with basis $X$, then the standard coaction $s_{X}: G \rightarrow \mathcal{E}_{f}$ is defined by $s_{X}(x)=$ $x f(x)=\eta_{x}$ for all $x \in X$.

We often abbreviate $D_{B}\left(s_{1}, s_{2}\right)$ to $D_{B}$ or simply $D$.

Proposition 6.3. Let $\Phi: f_{1} \rightarrow f_{2}$ be a map of homomorphisms, $G_{1}$ a free group with basis $B$ and $s_{2}: G_{2} \rightarrow \mathcal{E}_{f_{2}}$ a coaction. Then the following are equivalent:

(1) There exists a coaction $s_{1}: G_{1} \rightarrow \mathcal{E}_{f_{1}}$ compatible with $s_{2}$.

(2) There is a homomorphism $u: G_{1} \rightarrow\left[G_{1}, H_{1}\right]$ such that $\widetilde{\Phi} \circ u=$ $D_{B}\left(s_{B}, s_{2}\right)$.

(3) $D_{B}\left(s_{B}, s_{2}\right)\left(G_{1}\right) \subseteq \widetilde{\Phi}\left[G_{1}, H_{1}\right]=\left[\phi_{1}\left(G_{1}\right), \phi_{2}\left(H_{1}\right)\right]$.

In particular, if $\phi_{1}$ and $\phi_{2}$ are onto, then (1) holds.

Proof. $(1) \Rightarrow(2)$. Given $s_{1}: G_{1} \rightarrow \mathcal{E}_{f_{1}}$, define $u^{\prime}: G_{1} \rightarrow \mathcal{E}_{f_{1}}$ by

$$
u^{\prime}(b)=\left(b f_{1}(b)\right) s_{1}(\bar{b})
$$

for every $b \in B$. Then $p_{1} u^{\prime}=1$ and so there is a homomorphism $u: G_{1} \rightarrow$ $\left[G_{1}, H_{1}\right]$ such that $i_{1} u=u^{\prime}$. Clearly, $\widetilde{\Phi} \circ u=D_{B}$.

$(2) \Rightarrow(3)$. Obvious. 
$(3) \Rightarrow(1)$. We define a coaction $s_{1}$ rel $f_{1}$ as follows. For every $b \in B$, let $D_{B}(b)=\widetilde{\Phi}\left(e_{b}\right)$ for some $e_{b} \in\left[G_{1}, H_{1}\right]$. Then set

$$
s_{1}(b)=i_{1}\left(\bar{e}_{b}\right)\left(b f_{1}(b)\right) \text {. }
$$

It follows that $s_{1}$ is a coaction and $\Phi s_{1}=s_{2} \phi_{1}$.

Now we consider when a coaction $s_{1}$ as above induces a coaction $s_{2}$. As before, $\Phi: f_{1} \rightarrow f_{2}$. We need some restrictions on $\phi_{1}: G_{1} \rightarrow G_{2}$.

DeFinition 6.4. The homomorphism $\phi_{1}: G_{1} \rightarrow G_{2}$ of free groups with kernel $N_{1}$ and image $J_{1}$ is called free if disjoint sets $X, Y, \widehat{Y}$ and $Z$ can be found so that $X \cup Y$ is a basis of $G_{1}, \widehat{Y} \cup Z$ is a basis of $G_{2}, \phi_{1}(X)=1$, $\phi_{1} \mid Y: Y \rightarrow \widehat{Y}$ is a bijection and $J_{1}=\langle\widehat{Y}\rangle$. The quadruple $Q=(X, Y, \widehat{Y}, Z)$ is called a basis for $\phi_{1}$ (see [2, Definition 6.1]).

Note that $N_{1}$ is the normal closure of $\langle X\rangle$ and $G_{2}=J_{1} *\langle Z\rangle$. The following corollary to $[8$, Theorem 3.3] shows when a homomorphism is free.

Lemma 6.5. If $G_{1}$ and $G_{2}$ are free groups of finite rank, then $\phi_{1}: G_{1} \rightarrow$ $G_{2}$ is free if and only if $J_{1}=\operatorname{im} \phi_{1}$ is a free factor of $G_{2}$.

Now suppose $\Phi: f_{1} \rightarrow f_{2}$ is a map of homomorphisms and $\phi_{1}$ is free with basis $Q$. We investigate when a given coaction $s_{1}$ induces a coaction $s_{2}$. We consider the difference homomorphism $D=D_{X \cup Y}\left(s_{1}, s_{\widehat{Y} \cup Z}\right): G_{1} \rightarrow$ $\left[G_{2}, H_{2}\right]$.

Proposition 6.6. Let $\Phi: f_{1} \rightarrow f_{2}$ be a map of homomorphisms as above. Assume that $\phi_{1}$ is free with kernel $N_{1}$ and image $J_{1}$ and let $s_{1}$ : $G_{1} \rightarrow \mathcal{E}_{f_{1}}$ be a coaction. Then the following are equivalent:

(1) There exists a coaction $s_{2}: G_{2} \rightarrow \mathcal{E}_{f_{2}}$ induced by $s_{1}$.

(2) There exists a homomorphism $v: G_{2} \rightarrow\left[G_{2}, H_{2}\right]$ such that $v \circ \phi_{1}=D$.

(3) $s_{1}\left(N_{1}\right) \subseteq \operatorname{ker} \Phi$.

(4) $N_{1} \subseteq \operatorname{ker} D$.

Proof. (1) $\Rightarrow(2)$. Define $v^{\prime}: G_{2} \rightarrow \mathcal{E}_{f_{2}}$ by

$$
\begin{aligned}
& v^{\prime}(\widehat{y})=s_{2}(\widehat{y})\left(\widehat{y} f_{2}(\widehat{y})\right)^{-1} \quad \text { if } \widehat{y} \in \widehat{Y}, \\
& v^{\prime}(z)=1 \quad \text { if } z \in Z .
\end{aligned}
$$

Then $p_{2} v^{\prime}=1$, so $v^{\prime}$ induces $v: G_{2} \rightarrow\left[G_{2}, H_{2}\right]$ with $i_{2} v=v^{\prime}$. We show $v \phi_{1}=D$ by showing $v^{\prime} \phi_{1}=i_{2} D$ :

$$
v^{\prime} \phi_{1}(x)=1=s_{2} \phi_{1}(x)=\Phi s_{1}(x)=i_{2} D(x)
$$

and

$$
v^{\prime} \phi_{1}(y)=v^{\prime}(\widehat{y})=s_{2}(\widehat{y})\left(\widehat{y} f_{2}(\widehat{y})\right)^{-1}=\Phi s_{1}(y)\left(\widehat{y} f_{2}(\widehat{y})\right)^{-1}=i_{2} D(y) .
$$

$(2) \Rightarrow(3)$ and $(3) \Rightarrow(4)$ are obvious. 
$(4) \Rightarrow(1)$. Define $s_{2}$ by

$$
\begin{aligned}
& s_{2}(\widehat{y})=\left(i_{2} D\right)(y)\left(\widehat{y} f_{2}(\widehat{y})\right) \quad \text { if } \widehat{y} \in \widehat{Y}, \\
& s_{2}(z)=z f_{2}(z) \quad \text { if } z \in Z .
\end{aligned}
$$

By $(4), \Phi s_{1}(x)=i_{2} D(x)=1$ and so $s_{2} \phi_{1}(x)=1=\Phi s_{1}(x)$. Also,

$$
\begin{aligned}
s_{2} \phi_{1}(y) & =s_{2}(\widehat{y})=i_{2} D(y)\left(\widehat{y} f_{2}(\widehat{y})\right) \\
& =\Phi s_{1}(y)\left(\widehat{y} f_{2}(\widehat{y})\right)^{-1}\left(\widehat{y} f_{2}(\widehat{y})\right)=\Phi s_{1}(y) .
\end{aligned}
$$

Hence $s_{2}$ is compatible with $s_{1}$.

REMARKS 6.7. (1) Some of the implications of Proposition 6.6 hold under weaker hypotheses, e.g., $(3) \Rightarrow(1)$ can be proved assuming only that $G_{2}$ is free and that $J_{1}$ is a free factor of $G_{2}$. However, for simplicity we have made the blanket assumption that $\phi_{1}$ is a free homomorphism.

(2) We note that Proposition 6.6 implies [2, Proposition 5.3] which gives necessary and sufficient conditions for a coaction $s: G \rightarrow \mathcal{E}_{f}$ to induce a comultiplication on $H$.

\section{References}

[1] M. Arkowitz and M. Gutierrez, Comultiplications on free groups and wedges of circles, Trans. Amer. Math. Soc. 350 (1998), 1663-1680.

[2] - - - Coactions on groups, Proc. Roy. Soc. Edinburgh Sect. A 131 (2001), 225-239.

[3] G. Bergman, Supports of derivations, free factorizations and ranks of fixed subgroups in free groups, Trans. Amer. Math. Soc. 351 (1999), 1531-1550.

[4] W. Dicks and M. Dunwoody, On equalizers of sections, J. Algebra 216 (1999), 20-39.

[5] B. Eckmann and P. Hilton, Structure maps in group theory, Fund. Math. 50 (1961), 207-221.

[6] P. Hilton, Homotopy Theory and Duality, Gordon and Breach, 1965 .

[7] D. Kan, On monoids and their dual, Bol. Soc. Mat. Mexicana 3 (1958), 52-61.

[8] W. Magnus, A. Karrass and D. Solitar, Combinatorial Group Theory, Wiley, 1966.

[9] R. Switzer, Algebraic Topology-Homotopy and Homology, Springer, 1975.

Mathematics Department

Dartmouth College

Hanover, NH 03755, U.S.A.

E-mail: martin.arkowitz@dartmouth.edu
Mathematics Department

Tufts University

Medford, MA 02155, U.S.A.

E-mail: mgutierr@tufts.edu

Received 9 October 2000;

in revised form 16 August 2001 